CIRJE-F-728

\title{
New Unified Computational Algorithm in a High-Order Asymptotic Expansion Scheme
}

\author{
Kohta Takehara \\ Graduate School of Economics, University of Tokyo \\ Akihiko Takahashi \\ University of Tokyo \\ Masashi Toda \\ Graduate School of Economics, University of Tokyo \\ March 2010
}

CIRJE Discussion Papers can be downloaded without charge from:

http://www.e.u-tokyo.ac.jp/cirje/research/03research02dp.html

Discussion Papers are a series of manuscripts in their draft form. They are not intended for circulation or distribution except as indicated by the author. For that reason Discussion Papers may not be reproduced or distributed without the written consent of the author. 


\title{
New Unified Computational Algorithm in a High-Order Asymptotic Expansion Scheme *
}

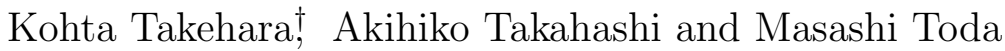 \\ Graduate School of Economics, the University of Tokyo \\ 7-3-1, Hongo, Bunkyo-ku, Tokyo, 113-0033, Japan \\ First Version: September 28, 2009 \\ Current Version: January 17, 2010 \\ Forthcoming in The Proceedings of KIER-TMU International Workshop on Financial
Engineering 2009
}

\begin{abstract}
An asymptotic expansion scheme in finance initiated by Kunitomo and Takahashi [6] and Yoshida [29] is a widely applicable methodology for analytic approximation of the expectation of a certain functional of diffusion processes. Mathematically, this methodology is justified by Watanabe theory([27]) in Malliavin calculus.

In practical applications, it is desirable to investigate the accuracy and stability of the method especially with expansion up to high orders in situations where the underlying processes are highly volatile as seen in the recent financial markets.

Although Takahashi[17], [18] and Takahashi and Takehara [20] provided explicit formulas for the expansion up to the third order, to our best knowledge a general computation scheme for an arbitraryorder expansion has not been given yet.

This paper proposes two general methods for computing the conditional expectations that are powerful especially for high order expansions: The first one, as an extension of the method introduced by the preceding papers, presents a unified scheme for computation of the conditional expectations. The second one develops a new calculation algorithm for computing the coefficients of the expansion through solving a system of ordinary differential equations that is equivalent to computing the conditional expectations.

To demonstrate their effectiveness, the paper gives numerical examples of the approximation for $\lambda$ SABR model up to the fifth order and a cross-currency Libor market model with a general stochastic volatility model of the spot foreign exchange rate up to the fourth order.
\end{abstract}

Keywords: Asymptotic Expansion, Malliavin Calculus, Approximation Formula, Stochastic Volatility, $\lambda$-SABR Model, Libor Market Model, Currency Options

AMS Subject Classifications: 91G80, 91G20, 60H07, 60H30, 60H35

\section{Introduction}

This paper presents two alternative schemes for computation in the method so-called "an asymptotic expansion approach" based on Watanabe theory(Watanabe [27]) in Malliavin calculus by extending the preceding papers and also by developing a new calculation algorithm.

${ }^{*}$ This research is partially supported by the global COE program "The research and training center for new development in mathematics" and Grant-in-Aid for JSPS Research Fellow.

†fin.tk.house@gmail.com. Research Fellow of the Japan Society for the Promotion of Science. 
To our best knowledge, the asymptotic expansion is first applied to finance for evaluation of an average option that is a popular derivative in commodity markets. [6] and [17] derive the approximation formulas for an average option by an asymptotic method based on log-normal approximations of an average price distribution when the underlying asset price follows a geometric Brownian motion. [29] applies a formula derived by the asymptotic expansion of certain statistical estimators for small diffusion processes. Thereafter, the asymptotic expansion have been applied to a broad class of problems in finance: See [18], [19], Kunitomo and Takahashi [7], [8], Matsuoka, Takahshi and Uchida [12], Takahashi and Yoshida [25], [26], Muroi [13], and Takahashi and Takehara [20], [21], [22].

For other asymptotic methods in finance which do not depend on Watanabe theory, see also Fouque, Papanicolaou and Sircar [3], [4], Henry-Labordere [10], [11], Kusuoka and Osajima [9], and Siopacha and Teichmann [16].

Recently, not only academic researchers but also many practitioners such as Antonov and Misirpashaev [1] or Andersen and Hutchings [2] have used the asymptotic expansion method based on Watanabe theory in their proposed techniques for a variety of financial issues. e.g. pricing or hedging complex derivatives under high-dimensional underlying stochastic environments. These methods are fully or partially based on the framework developed by [6], [17], [18] in a financial literature.

In theory, this method provides us the expansion of underlying stochastic processes which has a proper meaning in the limit of some ideal situations such as cases where they come deterministic ones (for details see [27], [28] or [8]).

In practice, however, we are often interested in cases far from that situation, where the underlying processes are highly volatile as seen in the recent financial markets especially after the crisis on 2008. Then from the view point of the accuracy or stability of the techniques in practical uses, it is desirable to investigate behaviors of its estimators in such situations especially with expansion up to high orders.

In the existing application of the asymptotic expansion based on Watanabe theory, they calculated certain conditional expectations which appear in their expansions and which play a key role in computation, by the formulas up to the third order given explicitly in [17], [18] and [20]. In many applications, these formulas give sufficiently accurate approximation, but in some cases, for example in the cases with long maturities and/or with highly volatile underlying variables, the approximation up to the third order may not provide satisfactory accuracies. Thus, the formulas for the higher order computation are desirable. But to our knowledge, asymptotic expansion formulas higher than the third order have not been given yet.

This paper provides the general procedures for the explicit computation of conditional expectations in the asymptotic expansion. Moreover, we develop an alternative but equivalent calculation algorithm which computes the unconditional expectations directly instead of the conditional ones and enables us to derive high order approximation formulas in an automatic manner. While these techniques can be applied to a broad class of Itô processes, for simplicity and limitation of space, in this paper we concentrate on a much simpler setting as described in Section 2. For further explanations in more general environment, see our online working paper Takahashi, Takehara and Toda [23].

Finally, our approximation generally shows sufficient accuracy with computation of high order expansions, which is confirmed by numerical experiments in more complex cases than that in Section 2.

Organization of this paper is as follows: After Section 2 will develop our methods in the simple setting, Section 3 applies our algorithms described in the previous section to the concrete financial models, and confirms the effectiveness of the higher order expansions by numerical examples in $\lambda$-SABR model and a cross-currency Libor market model with a general stochastic volatility model of the spot foreign exchange rate. Due to limitation of space, detailed proofs, the concrete expressions of some formulas and equations are omitted in this paper, which are given in [23]. We will refer to it if necessary.

\section{An Asymptotic Expansion Approach in a Black-Scholes Econ- omy}

In this section, our essential idea is explained in a simple Black-Scholes-type economy. For discussions in more general settings, refer to Section 3 and 4 of [23]. 


\subsection{An Asymptotic Expansion Approach in a Black-Scholes Economy}

Let $(W, P)$ be a one-dimensional Wiener space. Hereafter $P$ is considered as a risk-neutral equivalent martingale measure and a risk-free interest rate is set to be zero for simplicity. Then, the underlying economy is specified with a $\left(\mathbf{R}_{+}\right.$-valued)single risky asset $S^{(\epsilon)}=\left\{S_{t}^{(\epsilon)}\right\}$ satisfying

$$
S_{t}^{(\epsilon)}=S_{0}+\epsilon \int_{0}^{t} \sigma\left(S_{s}^{(\epsilon)}, s\right) d W_{s}
$$

where $\epsilon \in(0,1]$ is a constant parameter; $\sigma: \mathbf{R}_{+}^{2} \mapsto \mathbf{R}$ satisfies some regularity conditions. We will consider the following pricing problem;

$$
V(0, T)=\mathbf{E}\left[\Phi\left(S_{T}^{(\epsilon)}\right)\right]
$$

where $\Phi$ is a payoff function written on $S_{T}^{(\epsilon)}$ (for example, $\Phi(x)=\max (x-K, 0)$ for call options or $\Phi(x)=$ $\delta_{x}(x)$, a delta function with mass at $x$ for the density function) and $\mathbf{E}[\cdot]$ is an expectation operator under the probability measure $P$. Rigorously speaking, they are a generalized function on the Wiener functional $S^{(\epsilon)}$ and a generalized expectation defined for generalized functions respectively, whose mathematically proper definitions will be given in Section 2 of [23].

Let $A_{k t}=\left.\frac{\partial^{k} S_{t}^{(\epsilon)}}{\partial \epsilon^{k}}\right|_{\epsilon=0}$. Here we represent $A_{1 t}, A_{2 t}$ and $A_{3 t}$ explicitly by

$$
\begin{aligned}
& A_{1 t}=\int_{0}^{t} \sigma\left(S_{s}^{(0)}, s\right) d W_{s} \\
& A_{2 t}=2 \int_{0}^{t} \partial \sigma\left(S_{s}^{(0)}, s\right) A_{1 s} d W_{s} \\
& A_{3 t}=3 \int_{0}^{t}\left(\partial^{2} \sigma\left(S_{s}^{(0)}, s\right)\left(A_{1 s}\right)^{2}+\partial \sigma\left(S_{s}^{(0)}, s\right)\left(A_{2 s}\right)\right) d W_{s}
\end{aligned}
$$

recursively and then $S_{T}^{(\epsilon)}$ has its asymptotic expansion

$$
S_{T}^{(\epsilon)}=S_{0}+\epsilon A_{1 T}+\frac{\epsilon^{2}}{2 !} A_{2 T}+\frac{\epsilon^{3}}{3 !} A_{3 T}+o\left(\epsilon^{3}\right) .
$$

Note that $S_{t}^{(0)}=\lim _{\epsilon \downarrow 0} S_{t}^{(\epsilon)}=S_{0}$ for all $t$.

Next, normalize $S_{T}^{(\epsilon)}$ with respect to $\epsilon$ as

$$
G^{(\epsilon)}=\frac{S_{T}^{(\epsilon)}-S_{T}^{(0)}}{\epsilon}
$$

for $\epsilon \in(0,1]$. Then,

$$
G^{(\epsilon)}=A_{1 T}+\frac{\epsilon}{2 !} A_{2 T}+\frac{\epsilon^{2}}{3 !} A_{3 T}+o\left(\epsilon^{2}\right)
$$

in $L^{P}$ for every $p>1$. Here the following assumption is made:

$$
\Sigma_{T}=\int_{0}^{T} \sigma^{2}\left(S_{t}^{(0)}, t\right) d t>0 .
$$

Note that $A_{1 T}$ follows a normal distribution with mean 0 and variance $\Sigma_{T}$, and hence this assumption means that the distribution of $A_{1 T}$ does not degenerate. It is clear that this assumption is satisfied when $\sigma\left(S_{t}^{(0)}, t\right)>0$ for some $t>0$.

Then, the expectation of $\Phi\left(G^{(\epsilon)}\right)$ is expanded around $\epsilon=0$ up to $\epsilon^{2}$-order in the sense of Watanabe([27], Yoshida[28]) as follows (hereafter the asymptotic expansion of $\mathbf{E}\left[\Phi\left(G^{(\epsilon)}\right)\right]$ up to the second order will be considered):

$\mathbf{E}\left[\Phi\left(G^{(\epsilon)}\right)\right]=\mathbf{E}\left[\Phi\left(A_{1 T}\right)\right]+\epsilon \mathbf{E}\left[\Phi^{(1)}\left(A_{1 T}\right) A_{2 T}\right]$ 


$$
\begin{aligned}
& +\epsilon^{2}\left\{\mathbf{E}\left[\Phi^{(1)}\left(A_{1 T}\right) A_{3 T}\right]+\frac{1}{2} \mathbf{E}\left[\Phi^{(2)}\left(A_{1 T}\right)\left(A_{2 T}\right)^{2}\right]\right\}+o\left(\epsilon^{2}\right) \\
= & \mathbf{E}\left[\Phi\left(A_{1 T}\right)\right]+\epsilon \mathbf{E}\left[\Phi^{(1)}\left(A_{1 T}\right) \mathbf{E}\left[A_{2 T} \mid A_{1 T}\right]\right] \\
& +\epsilon^{2}\left\{\mathbf{E}\left[\Phi^{(1)}\left(A_{1 T}\right) \mathbf{E}\left[A_{3 T} \mid A_{1 T}\right]\right]+\frac{1}{2} \mathbf{E}\left[\Phi^{(2)}\left(A_{1 T}\right) \mathbf{E}\left[\left(A_{2 T}\right)^{2} \mid A_{1 T}\right]\right]\right\}+o\left(\epsilon^{2}\right) \\
= & \int_{\mathbf{R}} \Phi(x) f_{A_{1 T}}(x) d x+\epsilon \int_{\mathbf{R}} \Phi^{(1)}(x) \mathbf{E}\left[A_{2 T} \mid A_{1 T}=x\right] f_{A_{1 T}}(x) d x \\
& +\epsilon^{2}\left\{\int_{\mathbf{R}} \Phi^{(1)}(x) \mathbf{E}\left[A_{3 T} \mid A_{1 T}=x\right] f_{A_{1 T}}(x) d x+\frac{1}{2} \int_{\mathbf{R}} \Phi^{(2)}(x) \mathbf{E}\left[\left(A_{2 T}\right)^{2} \mid A_{1 T}=x\right] f_{A_{1 T}}(x) d x\right\}+o\left(\epsilon^{2}\right) \\
= & \int_{\mathbf{R}} \Phi(x) f_{A_{1 T}}(x) d x+\epsilon \int_{\mathbf{R}} \Phi(x)(-1) \frac{\partial}{\partial x}\left\{\mathbf{E}\left[A_{2 T} \mid A_{1 T}=x\right] f_{A_{1 T}}(x)\right\} d x \\
+ & \epsilon^{2}\left(\int_{\mathbf{R}} \Phi(x)(-1) \frac{\partial}{\partial x}\left\{\mathbf{E}\left[A_{3 T} \mid A_{1 T}=x\right] f_{A_{1 T}}(x)\right\} d x\right. \\
& \left.\quad+\frac{1}{2} \int_{\mathbf{R}} \Phi(x)(-1)^{2} \frac{\partial^{2}}{\partial x^{2}}\left\{\mathbf{E}\left[\left(A_{2 T}\right)^{2} \mid A_{1 T}=x\right] f_{A_{1 T}}(x)\right\} d x\right)+o\left(\epsilon^{2}\right)
\end{aligned}
$$

where $\Phi^{(m)}(x)$ is $m$-th order derivative of $\Phi(x)$ and $f_{A_{1 T}}(x)$ is a probability density function of $A_{1 T}$ following a normal distribution;

$$
f_{A_{1 T}}(x):=\frac{1}{\sqrt{2 \pi \Sigma_{T}}} \exp \left(-\frac{x^{2}}{2 \Sigma_{T}}\right) .
$$

In particular, letting $\Phi=\delta_{x}$, we have the asymptotic expansion of the density function of $G^{(\epsilon)}$ as seen later.

Then, all we have to do to evaluate this expansion is a computation of these conditional expectations. In particular, we present two alternative approaches.

\subsection{An Approach with an Expansion into Iterated Itô Integrals}

In this subsection we show an approach with a further expansion of $A_{2 T}, A_{3 T}$ and $\left(A_{2 T}\right)^{2}$ into iterated Itô integrals to compute the conditional expectations in (9).

Recall that we have

$$
\begin{aligned}
\mathbf{E}\left[\Phi\left(G^{(\epsilon)}\right)\right]= & \int_{\mathbf{R}} \Phi(x) f_{A_{1 T}}(x) d x+\epsilon \int_{\mathbf{R}} \Phi(x)(-1) \frac{\partial}{\partial x}\left\{\mathbf{E}\left[A_{2 T} \mid A_{1 T}=x\right] f_{A_{1 T}}(x)\right\} d x \\
+ & \epsilon^{2}\left(\int_{\mathbf{R}} \Phi(x)(-1) \frac{\partial}{\partial x}\left\{\mathbf{E}\left[A_{3 T} \mid A_{1 T}=x\right] f_{A_{1 T}}(x)\right\} d x\right. \\
& \left.+\frac{1}{2} \int_{\mathbf{R}} \Phi(x)(-1)^{2} \frac{\partial^{2}}{\partial x^{2}}\left\{\mathbf{E}\left[\left(A_{2 T}\right)^{2} \mid A_{1 T}=x\right] f_{A_{1 T}}(x)\right\} d x\right)+o\left(\epsilon^{2}\right) .
\end{aligned}
$$

Next, it is shown that $A_{2 T}, A_{3 T},\left(A_{2 T}\right)^{2}$ can be expressed as summations of iterated Itô integrals. First, note that $A_{2 T}$ is

$$
A_{2 T}=2 \int_{0}^{T} \int_{0}^{t_{1}} \partial \sigma\left(S_{t_{1}}^{(0)}, t_{1}\right) \sigma\left(S_{t_{2}}^{(0)}, t_{2}\right) d W_{t_{2}} d W_{t_{1}}
$$

Next, by application of Itô's formula to (5) we obtain

$$
\begin{aligned}
A_{3 T} & =6 \int_{0}^{T} \int_{0}^{t_{1}} \int_{0}^{t_{2}} \partial \sigma\left(S_{t_{1}}^{(0)}, t_{1}\right) \partial \sigma\left(S_{t_{2}}^{(0)}, t_{2}\right) \sigma\left(S_{t_{3}}^{(0)}, t_{3}\right) d W_{t_{3}} d W_{t_{2}} d W_{t_{1}} \\
& +6 \int_{0}^{T} \int_{0}^{t_{1}} \int_{0}^{t_{2}} \partial^{2} \sigma\left(S_{t_{1}}^{(0)}, t_{1}\right) \sigma\left(S_{t_{2}}^{(0)}, t_{2}\right) \sigma\left(S_{t_{3}}^{(0)}, t_{3}\right) d W_{t_{3}} d W_{t_{2}} d W_{t_{1}} \\
& +3 \int_{0}^{T} \int_{0}^{t_{1}} \partial^{2} \sigma\left(S_{t_{1}}^{(0)}, t_{1}\right) \sigma^{2}\left(S_{t_{2}}^{(0)}, t_{2}\right) d t_{2} d W_{t_{1}} .
\end{aligned}
$$


Similarly,

$$
\begin{aligned}
\left(A_{2 T}\right)^{2} & =16 \int_{0}^{T} \int_{0}^{t_{1}} \int_{0}^{t_{2}} \int_{0}^{t_{3}} \partial \sigma\left(S_{t_{1}}^{(0)}, t_{1}\right) \partial \sigma\left(S_{t_{2}}^{(0)}, t_{2}\right) \sigma\left(S_{t_{3}}^{(0)}, t_{3}\right) \sigma\left(S_{t_{4}}^{(0)}, t_{4}\right) d W_{t_{4}} d W_{t_{3}} d W_{t_{2}} d W_{t_{1}} \\
& +8 \int_{0}^{T} \int_{0}^{t_{1}} \int_{0}^{t_{2}} \int_{0}^{t_{3}} \partial \sigma\left(S_{t_{1}}^{(0)}, t_{1}\right) \sigma\left(S_{t_{2}}^{(0)}, t_{2}\right) \partial \sigma\left(S_{t_{3}}^{(0)}, t_{3}\right) \sigma\left(S_{t_{4}}^{(0)}, t_{4}\right) d W_{t_{4}} d W_{t_{3}} d W_{t_{2}} d W_{t_{1}} \\
& +8 \int_{0}^{T} \int_{0}^{t_{1}} \int_{0}^{t_{2}} \partial \sigma\left(S_{t_{1}}^{(0)}, t_{1}\right) \partial \sigma\left(S_{t_{2}}^{(0)}, t_{2}\right) \sigma^{2}\left(S_{t_{3}}^{(0)}, t_{3}\right) d t_{3} d W_{t_{2}} d W_{t_{1}} \\
& +8 \int_{0}^{T} \int_{0}^{t_{1}} \int_{0}^{t_{2}} \partial \sigma\left(S_{t_{1}}^{(0)}, t_{1}\right) \partial \sigma\left(S_{t_{2}}^{(0)}, t_{2}\right) \sigma\left(S_{t_{2}}^{(0)}, t_{2}\right) \sigma\left(S_{t_{3}}^{(0)}, t_{3}\right) d W_{t_{3}} d t_{2} d W_{t_{1}} \\
& +8 \int_{0}^{T} \int_{0}^{t_{1}} \int_{0}^{t_{2}}\left(\partial \sigma\left(S_{t_{1}}^{(0)}, t_{1}\right)\right)^{2} \sigma\left(S_{t_{2}}^{(0)}, t_{2}\right) \sigma\left(S_{t_{3}}^{(0)}, t_{3}\right) d W_{t_{3}} d W_{t_{2}} d t_{1} \\
& +4 \int_{0}^{T} \int_{0}^{t_{1}}\left(\partial \sigma\left(S_{t_{1}}^{(0)}, t_{1}\right)\right)^{2} \sigma^{2}\left(S_{t_{2}}^{(0)}, t_{2}\right) d t_{2} d t_{1} .
\end{aligned}
$$

Then, by Proposition 1 in [23], the conditional expectations in (11) can be computed as

$$
\begin{aligned}
\mathbf{E}\left[A_{2 T} \mid A_{1 T}=x\right] & =\left(2 \int_{0}^{T} \int_{0}^{t_{1}} \partial \sigma\left(S_{t_{1}}^{(0)}, t_{1}\right) \sigma\left(S_{t_{1}}^{(0)}, t_{1}\right) \sigma^{2}\left(S_{t_{2}}^{(0)}, t_{2}\right) d t_{2} d t_{1}\right) \frac{H_{2}\left(x ; \Sigma_{T}\right)}{\Sigma_{T}^{2}} \\
& =: \quad c_{2}^{2,1} H_{2}\left(x ; \Sigma_{T}\right) \\
\mathbf{E}\left[A_{3 T} \mid A_{1 T}=x\right] & =\left(6 \int_{0}^{T} \int_{0}^{t_{1}} \int_{0}^{t_{2}} \partial \sigma\left(S_{t_{1}}^{(0)}, t_{1}\right) \sigma\left(S_{t_{1}}^{(0)}, t_{1}\right) \partial \sigma\left(S_{t_{2}}^{(0)}, t_{2}\right) \sigma\left(S_{t_{2}}^{(0)}, t_{2}\right) \sigma^{2}\left(S_{t_{3}}^{(0)}, t_{3}\right) d t_{3} d t_{2} d t_{1}\right. \\
& \left.+6 \int_{0}^{T} \int_{0}^{t_{1}} \int_{0}^{t_{2}} \partial^{2} \sigma\left(S_{t_{1}}^{(0)}, t_{1}\right) \sigma\left(S_{t_{1}}^{(0)}, t_{1}\right) \sigma^{2}\left(S_{t_{2}}^{(0)}, t_{2}\right) \sigma^{2}\left(S_{t_{3}}^{(0)}, t_{3}\right) d t_{3} d t_{2} d t_{1}\right) \frac{H_{3}\left(x ; \Sigma_{T}\right)}{\Sigma_{T}^{3}} \\
& +\left(3 \int_{0}^{T} \int_{0}^{t_{1}} \partial^{2} \sigma\left(S_{t_{1}}^{(0)}, t_{1}\right) \sigma\left(S_{t_{1}}^{(0)}, t_{1}\right) \sigma^{2}\left(S_{t_{2}}^{(0)}, t_{2}\right) d t_{2} d t_{1}\right) \frac{H_{1}\left(x ; \Sigma_{T}\right)}{\Sigma_{T}} \\
& =: c_{3}^{3,1} H_{3}\left(x ; \Sigma_{T}\right)+c_{1}^{3,1} H_{1}\left(x ; \Sigma_{T}\right)
\end{aligned}
$$

and

$$
\begin{aligned}
& \mathbf{E}\left[\left(A_{2 T}\right)^{2} \mid A_{1 T}=x\right] \\
= & \left(16 \int_{0}^{T} \int_{0}^{t_{1}} \int_{0}^{t_{2}} \int_{0}^{t_{3}} \partial \sigma\left(S_{t_{1}}^{(0)}, t_{1}\right) \sigma\left(S_{t_{1}}^{(0)}, t_{1}\right) \partial \sigma\left(S_{t_{2}}^{(0)}, t_{2}\right) \sigma\left(S_{t_{2}}^{(0)}, t_{2}\right) \sigma^{2}\left(S_{t_{3}}^{(0)}, t_{3}\right) \sigma^{2}\left(S_{t_{4}}^{(0)}, t_{4}\right) d t_{4} d t_{3} d t_{2} d t_{1}\right. \\
+ & \left.8 \int_{0}^{T} \int_{0}^{t_{1}} \int_{0}^{t_{2}} \int_{0}^{t_{3}} \partial \sigma\left(S_{t_{1}}^{(0)}, t_{1}\right) \sigma\left(S_{t_{1}}^{(0)}, t_{1}\right) \sigma^{2}\left(S_{t_{2}}^{(0)}, t_{2}\right) \partial \sigma\left(S_{t_{3}}^{(0)}, t_{3}\right) \sigma\left(S_{t_{3}}^{(0)}, t_{3}\right) \sigma^{2}\left(S_{t_{4}}^{(0)}, t_{4}\right) d t_{4} d t_{3} d t_{2} d t_{1}\right) \frac{H_{4}\left(x ; \Sigma_{T}\right)}{\Sigma_{T}^{4}} \\
+ & \left(16 \int_{0}^{T} \int_{0}^{t_{1}} \int_{0}^{t_{2}} \partial \sigma\left(S_{t_{1}}^{(0)}, t_{1}\right) \sigma\left(S_{t_{1}}^{(0)}, t_{1}\right) \partial \sigma\left(S_{t_{2}}^{(0)}, t_{2}\right) \sigma\left(S_{t_{2}}^{(0)}, t_{2}\right) \sigma^{2}\left(S_{t_{3}}^{(0)}, t_{3}\right) d t_{3} d t_{2} d t_{1}\right. \\
+ & \left.8 \int_{0}^{T} \int_{0}^{t_{1}} \int_{0}^{t_{2}}\left(\partial \sigma\left(S_{t_{1}}^{(0)}, t_{1}\right)\right)^{2} \sigma^{2}\left(S_{t_{2}}^{(0)}, t_{2}\right) \sigma^{2}\left(S_{t_{3}}^{(0)}, t_{3}\right) d t_{3} d t_{2} d t_{1}\right) \frac{H_{2}\left(x ; \Sigma_{T}\right)}{\Sigma_{T}^{2}} \\
+ & \left(4 \int_{0}^{T} \int_{0}^{t_{1}}\left(\partial \sigma\left(S_{t_{1}}^{(0)}, t_{1}\right)\right)^{2} \sigma^{2}\left(S_{t_{2}}^{(0)}, t_{2}\right) d t_{2} d t_{1}\right) H_{0}\left(x ; \Sigma_{T}\right) \\
=: & c_{4}^{2,2} H_{4}\left(x ; \Sigma_{T}\right)+c_{2}^{2,2} H_{2}\left(x ; \Sigma_{T}\right)+c_{0}^{2,2} H_{0}\left(x ; \Sigma_{T}\right)
\end{aligned}
$$


where $H_{n}(x ; \Sigma)$ is an $n$-th order Hermite polynomial defined by

$$
H_{n}(x ; \Sigma):=(-\Sigma)^{n} e^{x^{2} / 2 \Sigma} \frac{d^{n}}{d x^{n}} e^{-x^{2} / 2 \Sigma} .
$$

Substituting these into (11), we have the asymptotic expansion of $\mathbf{E}\left[\Phi\left(G^{(\epsilon)}\right)\right]$ up to $\epsilon^{2}$-order. Further, letting $\Phi=\delta_{x}$, we have the expansion of $f_{G^{(\epsilon)}}$, the density function of $G^{(\epsilon)}$ :

$$
\begin{aligned}
f_{G^{(\epsilon)}} & =f_{A_{1 T}}(x)+\epsilon(-1) \frac{\partial}{\partial x}\left\{\mathbf{E}\left[A_{2 T} \mid A_{1 T}=x\right] f_{A_{1 T}}(x)\right\} \\
& +\epsilon^{2}\left((-1) \frac{\partial}{\partial x}\left\{\mathbf{E}\left[A_{3 T} \mid A_{1 T}=x\right] f_{A_{1 T}}(x)\right\}+\frac{1}{2}(-1)^{2} \frac{\partial^{2}}{\partial x^{2}}\left\{\mathbf{E}\left[\left(A_{2 T}\right)^{2} \mid A_{1 T}=x\right] f_{A_{1 T}}(x)\right\}\right)+o\left(\epsilon^{2}\right) \\
& =f_{A_{1 T}}(x)+\epsilon(-1) \frac{\partial}{\partial x}\left\{c_{2}^{2,1} H_{2}\left(x ; \Sigma_{T}\right) f_{A_{1 T}}(x)\right\} \\
& +\epsilon^{2}\left((-1) \frac{\partial}{\partial x}\left\{\sum_{i=1,3} c_{i}^{3,1} H_{i}\left(x ; \Sigma_{T}\right) f_{A_{1 T}}(x)\right\}+\frac{1}{2}(-1)^{2} \frac{\partial^{2}}{\partial x^{2}}\left\{\sum_{i=0,2,4} c_{i}^{2,2} H_{i}\left(x ; \Sigma_{T}\right) f_{A_{1 T}}(x)\right\}\right)+o\left(\epsilon^{2}\right) .
\end{aligned}
$$

\subsection{An Alternative Approach with a System of Ordinary Differential Equations}

In this subsection, we present an alternative approachin which the conditional expectations are computed through some system of ordinary differential equations.

Again the asymptotic expansion of $\mathbf{E}\left[\Phi\left(G^{(\epsilon)}\right)\right]$ up to $\epsilon^{2}$-order is considered in this subsection.

Note that the expectations of $A_{2 T}, A_{3 T}$ and $\left(A_{2 T}\right)^{2}$ conditional on $A_{1 T}$ are expressed by linear combinations of a finite number of Hermite polynomials as in (15), (16) and (17). Thus, by Lemma 4 in [23], we have

$$
\begin{aligned}
\mathbf{E}\left[A_{2 T} \mid A_{1 T}=x\right] & =\sum_{n=0}^{2} a_{n}^{2,1} H_{n}\left(x ; \Sigma_{T}\right), \\
\mathbf{E}\left[A_{3 T} \mid A_{1 T}=x\right] & =\sum_{n=0}^{3} a_{n}^{3,1} H_{n}\left(x ; \Sigma_{T}\right), \\
\text { and } \mathbf{E}\left[\left(A_{2 T}\right)^{2} \mid A_{1 T}=x\right] & =\sum_{n=0}^{4} a_{n}^{2,2} H_{n}\left(x ; \Sigma_{T}\right),
\end{aligned}
$$

where the coefficients are given by

$$
\begin{aligned}
a_{n}^{2,1} & =\left.\frac{1}{n !} \frac{1}{(i \Sigma)^{n}} \frac{\partial^{n}}{\partial \xi^{n}}\right|_{\xi=0}\left\{\mathbf{E}\left[Z_{T}^{\langle\xi\rangle} A_{2 T}\right]\right\}, \\
a_{n}^{3,1} & =\left.\frac{1}{n !} \frac{1}{(i \Sigma)^{n}} \frac{\partial^{n}}{\partial \xi^{n}}\right|_{\xi=0}\left\{\mathbf{E}\left[Z_{T}^{\langle\xi\rangle} A_{3 T}\right]\right\}, \\
a_{n}^{2,2} & =\left.\frac{1}{n !} \frac{1}{(i \Sigma)^{n}} \frac{\partial^{n}}{\partial \xi^{n}}\right|_{\xi=0}\left\{\mathbf{E}\left[Z_{T}^{\langle\xi\rangle}\left(A_{2 T}\right)^{2}\right]\right\}, \\
\text { and } Z_{t}^{\langle\xi\rangle} & :=\exp \left(i \xi A_{1 t}+\frac{\xi^{2}}{2} \Sigma_{t}\right) .
\end{aligned}
$$

Note that $Z^{\langle\xi\rangle}$ is a martingale with $Z_{0}^{\langle\xi\rangle}=1$. Since these conditional expectations can be represented by linear combinations of Hermite polynomials as seen in the previous subsection, the following should hold, 
which can be confirmed easily with results of this subsection:

$$
\left\{\begin{array}{l}
a_{2}^{2,1}=c_{2}^{2,1} ; \quad a_{1}^{2,1}=a_{0}^{2,1}=0 ; \\
a_{3}^{3,1}=c_{3}^{3,1} ; \quad a_{1}^{3,1}=c_{1}^{3,1} ; \quad a_{2}^{3,1}=a_{0}^{2,1}=0 ; \\
a_{4}^{2,2}=c_{4}^{2,2} ; \quad a_{2}^{2,2}=c_{2}^{2,2} ; \quad a_{0}^{2,2}=c_{0}^{2,2} ; \quad a_{3}^{2,2}=a_{1}^{2,2}=0 .
\end{array}\right.
$$

Then, computation of these conditional expectations is equivalent to that of the unconditional expectations $\mathbf{E}\left[Z_{T}^{\langle\xi\rangle} A_{2 T}\right], \mathbf{E}\left[Z_{T}^{\langle\xi\rangle} A_{3 T}\right]$ and $\mathbf{E}\left[Z_{T}^{\langle\xi\rangle}\left(A_{2 T}\right)^{2}\right]$.

First, applying Itô's formula to $\left(Z_{t}^{\langle\xi\rangle} A_{2 t}\right)$ we have

$$
\begin{aligned}
\mathbf{E}\left[Z_{t}^{\langle\xi\rangle} A_{2 t}\right] & =\mathbf{E}\left[\int_{0}^{t} Z_{s}^{\langle\xi\rangle} d A_{2 s}+\int_{0}^{t} A_{2 s} d Z_{s}^{\langle\xi\rangle}+\left\langle A_{2}, Z^{\langle\xi\rangle}\right\rangle_{t}\right] \\
& =2(i \xi) \int_{0}^{t} \partial \sigma\left(S_{s}^{(0)}, s\right) \sigma\left(S_{s}^{(0)}, s\right) \mathbf{E}\left[Z_{s}^{\langle\xi\rangle} A_{1 s}\right] d s
\end{aligned}
$$

Then, applying Itô's formula to $\left(Z_{t}^{\langle\xi\rangle} A_{1 t}\right)$ again, we also have

$$
\begin{aligned}
\mathbf{E}\left[Z_{t}^{\langle\xi\rangle} A_{1 t}\right] & =\mathbf{E}\left[\int_{0}^{t} Z_{s}^{\langle\xi\rangle} d A_{1 s}+\int_{0}^{t} A_{1 s} d Z_{s}^{\langle\xi\rangle}+\left\langle A_{1}, Z^{\langle\xi\rangle}\right\rangle_{t}\right] \\
& =(i \xi) \int_{0}^{t} \sigma^{2}\left(S_{s}^{(0)}, s\right) \mathbf{E}\left[Z_{s}^{\langle\xi\rangle}\right] d s \\
& =(i \xi) \int_{0}^{t} \sigma^{2}\left(S_{s}^{(0)}, s\right) d s
\end{aligned}
$$

since $\mathbf{E}\left[Z_{t}^{\langle\xi\rangle}\right]=1$ for all $t$.

Similarly, the followings are obtained;

$$
\begin{aligned}
\mathbf{E}\left[Z_{t}^{\langle\xi\rangle} A_{3 t}\right]= & 3(i \xi)\left(\int_{0}^{t} \partial^{2} \sigma\left(S_{s}^{(0)}, s\right) \sigma\left(S_{s}^{(0)}, s\right) \mathbf{E}\left[Z_{s}^{\langle\xi\rangle}\left(A_{1 s}\right)^{2}\right] d s\right. \\
& \left.+\int_{0}^{t} \partial \sigma\left(S_{s}^{(0)}, s\right) \sigma\left(S_{s}^{(0)}, s\right) \mathbf{E}\left[Z_{s}^{\langle\xi\rangle} A_{2 s}\right] d s\right) \\
\mathbf{E}\left[Z_{t}^{\langle\xi\rangle}\left(A_{1 t}\right)^{2}\right]= & \int_{0}^{t} \sigma^{2}\left(S_{s}^{(0)}, s\right) d s \\
& +2(i \xi) \int_{0}^{t} \sigma^{2}\left(S_{s}^{(0)}, s\right) \mathbf{E}\left[Z_{s}^{\langle\xi\rangle} A_{1 s}\right] d s \\
\mathbf{E}\left[Z_{t}^{\langle\xi\rangle}\left(A_{2 t}\right)^{2}\right]= & 4 \int_{0}^{t}\left(\partial \sigma\left(S_{s}^{(0)}, s\right)\right)^{2} \mathbf{E}\left[Z_{s}^{\langle\xi\rangle}\left(A_{1 s}\right)^{2}\right] d s \\
& +4(i \xi) \int_{0}^{t} \partial \sigma\left(S_{s}^{(0)}, s\right) \sigma\left(S_{s}^{(0)}, s\right) \mathbf{E}\left[Z_{s}^{\langle\xi\rangle} A_{2 s} A_{1 s}\right] d s \\
\mathbf{E}\left[Z_{t}^{\langle\xi\rangle} A_{2 t} A_{1 t}\right]= & 2 \int_{0}^{t} \partial \sigma\left(S_{s}^{(0)}, s\right) \sigma\left(S_{s}^{(0)}, s\right) \mathbf{E}\left[Z_{s}^{\langle\xi\rangle} A_{1 s}\right] d s \\
& +(1 \xi) \int_{0}^{t}\left(\sigma\left(S_{s}^{(0)}, s\right)\right)^{2} \mathbf{E}\left[Z_{s}^{\langle\xi\rangle} A_{2 s}\right] d s \\
& +2(1 \xi) \int_{0}^{t} \partial \sigma\left(S_{s}^{(0)}, s\right) \sigma\left(S_{s}^{(0)}, s\right) \mathbf{E}\left[Z_{s}^{\langle\xi\rangle}\left(A_{1 s}\right)^{2}\right] d s .
\end{aligned}
$$


Then, $\mathbf{E}\left[Z_{T}^{\langle\xi\rangle} A_{2 T}\right], \mathbf{E}\left[Z_{T}^{\langle\xi\rangle} A_{3 T}\right]$ and $\mathbf{E}\left[Z_{T}^{\langle\xi\rangle}\left(A_{2 T}\right)^{2}\right]$ can be obtained as solutions of the system of ordinary differential equations $(23),(24),(25),(26),(27)$ and $(28)$. In fact, since they have a grading structure that the higher-order equations depend only on the lower ones as

$$
\begin{aligned}
& \mathbf{E}\left[Z_{t}^{\langle\xi\rangle} A_{1 t}\right]=(i \xi) \int_{0}^{t} \sigma^{2}\left(S_{s}^{(0)}, s\right) d s \\
& \mathbf{E}\left[Z_{t}^{\langle\xi\rangle} A_{2 t}\right]=2(i \xi) \int_{0}^{t} \partial \sigma\left(S_{s}^{(0)}, s\right) \sigma\left(S_{s}^{(0)}, s\right) \mathbf{E}\left[Z_{s}^{\langle\xi\rangle} A_{1 s}\right] d s \\
& \mathbf{E}\left[Z_{t}^{\langle\xi\rangle}\left(A_{1 t}\right)^{2}\right]=\int_{0}^{t} \sigma^{2}\left(S_{s}^{(0)}, s\right) d s \\
& +2(i \xi) \int_{0}^{t} \sigma^{2}\left(S_{s}^{(0)}, s\right) \mathbf{E}\left[Z_{s}^{\langle\xi\rangle} A_{1 s}\right] d s \\
& \mathbf{E}\left[Z_{t}^{\langle\xi\rangle} A_{3 t}\right]=3(i \xi)\left(\int_{0}^{t} \partial^{2} \sigma\left(S_{s}^{(0)}, s\right) \sigma\left(S_{s}^{(0)}, s\right) \mathbf{E}\left[Z_{s}^{\langle\xi\rangle}\left(A_{1 s}\right)^{2}\right] d s\right. \\
& \left.+\int_{0}^{t} \partial \sigma\left(S_{s}^{(0)}, s\right) \sigma\left(S_{s}^{(0)}, s\right) \mathbf{E}\left[Z_{s}^{\langle\xi\rangle} A_{2 s}\right] d s\right) \\
& \mathbf{E}\left[Z_{t}^{\langle\xi\rangle} A_{2 t} A_{1 t}\right]=2 \int_{0}^{t} \partial \sigma\left(S_{s}^{(0)}, s\right) \sigma\left(S_{s}^{(0)}, s\right) \mathbf{E}\left[Z_{s}^{\langle\xi\rangle} A_{1 s}\right] d s \\
& +(i \xi) \int_{0}^{t}\left(\sigma\left(S_{s}^{(0)}, s\right)\right)^{2} \mathbf{E}\left[Z_{s}^{\langle\xi\rangle} A_{2 s}\right] d s \\
& +2(i \xi) \int_{0}^{t} \partial \sigma\left(S_{s}^{(0)}, s\right) \sigma\left(S_{s}^{(0)}, s\right) \mathbf{E}\left[Z_{s}^{\langle\xi\rangle}\left(A_{1 s}\right)^{2}\right] d s \\
& \mathbf{E}\left[Z_{t}^{\langle\xi\rangle}\left(A_{2 t}\right)^{2}\right]=4 \int_{0}^{t}\left(\partial \sigma\left(S_{s}^{(0)}, s\right)\right)^{2} \mathbf{E}\left[Z_{s}^{\langle\xi\rangle}\left(A_{1 s}\right)^{2}\right] d s \\
& +4(i \xi) \int_{0}^{t} \partial \sigma\left(S_{s}^{(0)}, s\right) \sigma\left(S_{s}^{(0)}, s\right) \mathbf{E}\left[Z_{s}^{\langle\xi\rangle} A_{2 s} A_{1 s}\right] d s,
\end{aligned}
$$

they can be easily solved with substituting each solution into the next ordinary differential equation recursively. Moreover, since these solutions are clearly the polynomial of $(i \xi)$, we can easily implement differentiations with respect to $\xi$ in (19), (20) and (21). It is obvious that the resulting coefficients given by these solutions are equivalent to the results in the previous subsection.

Moreover, we also remark the relationship between our method and an approach presented by [18] in which the density function of $G^{(\epsilon)}$ is derived by Fourier inversion of its formally expanded characteristic function. Precisely speaking, [18] formally expanded $\Psi_{G^{(\epsilon)}}(\xi)=\mathbf{E}\left[e^{i \xi G^{(\epsilon)}}\right]$ as

$$
\begin{aligned}
& \Psi_{G^{(\epsilon)}}(\xi)=\mathbf{E}\left[e^{i \xi G^{(\epsilon)}}\right]=e^{-\frac{\xi^{2}}{2} \Sigma_{T}} \times\left\{1+\epsilon(i \xi) \mathbf{E}\left[Z_{T}^{\langle\xi\rangle} A_{2 T}\right]+\epsilon^{2}\left((i \xi) \mathbf{E}\left[Z_{T}^{\langle\xi\rangle} A_{3 T}\right]+\frac{(i \xi)^{2}}{2} \mathbf{E}\left[Z_{T}^{\langle\xi\rangle}\left(A_{2 T}\right)^{2}\right]\right)\right\}+o\left(\epsilon^{2}\right) \\
&= e^{-\frac{\xi^{2}}{2} \Sigma_{T}} \times\left\{1+\epsilon(i \xi) \mathbf{E}\left[Z_{T}^{\langle\xi\rangle} \mathbf{E}\left[A_{2 T} \mid A_{1 T}\right]\right]\right. \\
&\left.\epsilon^{2}\left((i \xi) \mathbf{E}\left[Z_{T}^{\langle\xi\rangle} \mathbf{E}\left[A_{3 T} \mid A_{1 T}\right]\right]+\frac{(i \xi)^{2}}{2} \mathbf{E}\left[Z_{T}^{\langle\xi\rangle} \mathbf{E}\left[\left(A_{2 T}\right)^{2} \mid A_{1 T}\right]\right]\right)\right\}+o\left(\epsilon^{2}\right)
\end{aligned}
$$

and computed the conditional expectations in this expansion. Then, $f_{G^{(\epsilon)}}(x)$, the density function of $G^{(\epsilon)}$, was derived by Fourier inversion of $\Psi_{G^{(\epsilon)}}(\xi)$;

$$
f_{G^{(\epsilon)}}(x)=\mathcal{F}^{-1}\left(\Psi_{G^{(\epsilon)}}\right)=\frac{1}{2 \pi} \int_{-\infty}^{\infty} e^{-i x \xi} \Psi_{G^{(\epsilon)}}(\xi) d \xi
$$

This approach is completely equivalent to our method based on Watanabe theory as also mentioned in 
[18]. In fact, from (18) and (22) we obtain

$$
\begin{aligned}
f_{G^{(\epsilon)}}(x)= & f_{A_{1 T}}(x)+\epsilon(-1) \frac{\partial}{\partial x}\left\{c_{2}^{2,1} H_{2}\left(x ; \Sigma_{T}\right) f_{A_{1 T}}(x)\right\} \\
+ & \epsilon^{2}\left((-1) \frac{\partial}{\partial x}\left\{\sum_{n=1,3} c_{n}^{3,1} H_{n}\left(x ; \Sigma_{T}\right) f_{A_{1 T}}(x)\right\}+\frac{1}{2}(-1)^{2} \frac{\partial^{2}}{\partial x^{2}}\left\{\sum_{n=0,2,4} c_{n}^{2,2} H_{n}\left(x ; \Sigma_{T}\right) f_{A_{1 T}}(x)\right\}\right)+o\left(\epsilon^{2}\right) \\
= & \mathcal{F}^{-1}\left(e^{-\frac{\xi^{2}}{2} \Sigma_{T}}\right)+\epsilon c_{2}^{2,1} \mathcal{F}^{-1}\left((i \xi)\left(i \xi \Sigma_{T}\right)^{2} e^{-\frac{\xi^{2}}{2} \Sigma_{T}}\right) \\
+ & \epsilon^{2}\left(\sum_{n=1,3} c_{n}^{3,1} \mathcal{F}^{-1}\left((i \xi)\left(i \xi \Sigma_{T}\right)^{n} e^{-\frac{\xi^{2}}{2} \Sigma_{T}}\right)+\frac{1}{2} \sum_{n=0,2,4} c_{n}^{2,2} \mathcal{F}^{-1}\left((i \xi)^{2}\left(i \xi \Sigma_{T}\right)^{n} e^{-\frac{\xi^{2}}{2} \Sigma_{T}}\right)\right)+o\left(\epsilon^{2}\right) \\
= & \mathcal{F}^{-1}\left(e^{-\frac{\xi^{2}}{2} \Sigma_{T}} \times\left\{1+\epsilon(i \xi) \sum_{n=0}^{2} a_{n}^{2,1}\left(i \Sigma_{T}\right)^{n} \xi^{n}\right.\right. \\
& \left.\left.\quad \epsilon^{2}\left((i \xi) \sum_{n=0}^{3} a_{n}^{3,1}\left(i \Sigma_{T}\right)^{n} \xi^{n}+\frac{(i \xi)^{2}}{2} \sum_{n=0}^{4} a_{n}^{2,2}\left(i \Sigma_{T}\right)^{n} \xi^{n}\right)\right\}\right)+o\left(\epsilon^{2}\right) \\
= & \mathcal{F}^{-1}\left(e^{-\frac{\xi^{2}}{2} \Sigma_{T}} \times\left\{1+\epsilon(i \xi) \mathbf{E}\left[Z_{T}^{\langle\xi\rangle} A_{2 T}\right]\right.\right. \\
& \left.\left.\quad \epsilon^{2}\left((i \xi) \mathbf{E}\left[Z_{T}^{\langle\xi\rangle} A_{3 T}\right]+\frac{(i \xi)^{2}}{2} \mathbf{E}\left[Z_{T}^{\langle\xi\rangle}\left(A_{2 T}\right)^{2}\right]\right)\right\}\right)+o\left(\epsilon^{2}\right) .
\end{aligned}
$$

Then it is obvious that the inversion of the characteristic function expanded up to $\epsilon^{2}$-order (29) coincides with the density function obtained by our approach. Moreover, it can be shown that this equivalence holds at any order.

Here, at the end of this section, we state a brief summary. In the Black-Scholes-type economy, we consider the risky asset $S^{(\epsilon)}$ and evaluate some quantities, expressed as an expectation of the function of the future price, such as prices or risk sensitivities of the securities on this asset. First we expand them around the limit to $\epsilon=0$ so that we obtain the expansion (9) which contains some conditional expectations. Then, by approaches described in Section 2.2 or 2.3, we compute these conditional expectation. Finally, substituting computation results into (9), we obtain the asymptotic expansion of those quantities. Or equivalently, one can use the formulas for these conditional expectations listed up in [23].

\section{$3 \quad$ Numerical Examples}

In this section we apply the proposed techniques to the model more complex than Black-Scholes-type case in the previous section, to demonstrate their effectiveness. Detailed discussions in a general setting including following examples are found in Section 3 and 4 of [23].

\section{$3.1 \quad \lambda$-SABR model}

We first consider the European plain-vanilla call and put prices under the following $\lambda$-SABR model [10] (interest rate $=0 \%$ ) :

$$
\begin{aligned}
& d S^{(\epsilon)}(t)=\epsilon \sigma^{(\epsilon)}(t)\left(S^{(\epsilon)}(t)\right)^{\beta} d W_{t}^{1} \\
& d \sigma^{(\epsilon)}(t)=\lambda\left(\theta-\sigma^{(\epsilon)}(t)\right) d t+\epsilon \nu_{1} \sigma^{(\epsilon)}(t) d W_{t}^{1}+\epsilon \nu_{2} \sigma^{(\epsilon)}(t) d W_{t}^{2},
\end{aligned}
$$

where $\nu_{1}=\rho \nu, \nu_{2}=\left(\sqrt{1-\rho^{2}}\right) \nu$. (The correlation between $S$ and $\sigma$ is $\rho \in[-1,1]$.) 
Table 1: Parameter specifications of the $\lambda$-SABR model for our numerical experiments.

\begin{tabular}{|c|c|c|c|c|c|c|c|c|}
\hline Parameter & $S(0)$ & $\lambda$ & $\sigma(0)$ & $\beta$ & $\rho$ & $\theta$ & $\nu$ & $T$ \\
\hline$\overline{\mathrm{i}}$ & 100 & $\overline{0.1}$ & 3.0 & 0.5 & -0.7 & 3.0 & $\overline{0.3}$ & $\overline{10}$ \\
\hline ii & 100 & 0.1 & 0.3 & 1.0 & -0.7 & 0.3 & 0.3 & 10 \\
\hline iii & 100 & 0.1 & 0.3 & 1.0 & -0.7 & 0.3 & 0.3 & 30 \\
\hline
\end{tabular}

Approximated prices by the asymptotic expansion method are calculated up to the fifth order. Note that all the solutions to differential equations are obtained analytically. Benchmark values are computed by Monte Carlo simulations. $\epsilon$ is set to be one and other parameters used in the test are given in Table 1:

For the case of $\beta=1$ (i.e. case ii and iii), we calculate approximated prices by the "log-normal asymptotic expansion method" described in Section 4.3 in [23] up to the fourth order. In Monte Carlo simulations for benchmark values, we use Euler-Maruyama scheme as a discretization scheme with 1024 time steps for case $\mathrm{i}$ and for case ii and iii the second order discretization scheme given by Ninomiya-Victoir [14] with 128 and 256 time steps, respectively. Each simulation contains $10^{8}$ paths.

Results are in Table 2.

From the results, in each case, the higher order asymptotic expansion or log-normal asymptotic expansion almost always improve the accuracy of approximation by the lower expansions. Improvement is significant especially in long-term cases in which the lower order asymptotic expansions cannot approximate the price well. 


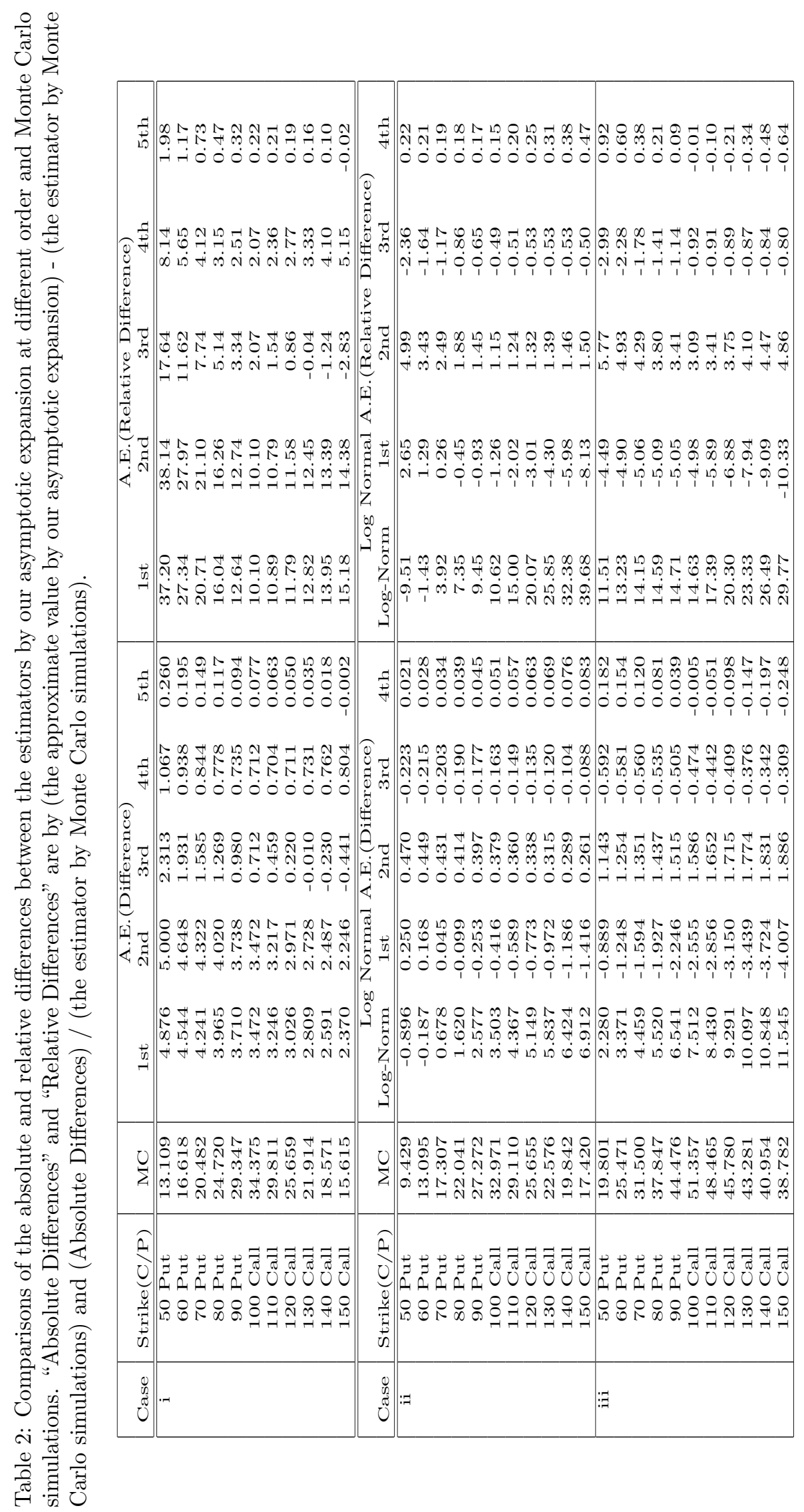




\subsection{Currency Option under a Libor Market Model of Interest Rates and a Stochastic Volatility of a Spot Exchange Rate}

In this subsection, we apply our methods to pricing options on currencies under Libor Market Models(LMMs) of interest rates and a stochastic volatility of the spot foreign exchange rate(Forex). Due to limitation of space, only the structure of the stochastic differential equations of our model is described here. For details of the underlying model, see Takahashi and Takehara [20].

\subsubsection{Cross-Currency Libor Market Models}

Let $\left(\Omega, \mathcal{F}, \tilde{P},\left\{\mathcal{F}_{t}\right\}_{0 \leq t \leq T^{*}<\infty}\right)$ be a complete probability space with a filtration satisfying the usual conditions. We consider the following pricing problem for the call option with maturity $T \in\left(0, T^{*}\right]$ and strike rate $K>0$;

$$
V^{C}(0 ; T, K)=P_{d}(0, T) \times \mathbf{E}^{P}\left[(S(T)-K)^{+}\right]=P_{d}(0, T) \times \mathbf{E}^{P}\left[\left(F_{T}(T)-K\right)^{+}\right]
$$

where $V^{C}(0 ; T, K)$ denotes the value of an European call option at time 0 with maturity $T$ and strike rate $K, S(T)$ denotes the spot exchange rate at time $t \geq 0$ and $F_{T}(t)$ denotes the time $t$ value of the forex forward rate with maturity $T$. Similarly, for the put option we consider

$$
V^{P}(0 ; T, K)=P_{d}(0, T) \times \mathbf{E}^{P}\left[(K-S(T))^{+}\right]=P_{d}(0, T) \times \mathbf{E}^{P}\left[\left(K-F_{T}(T)\right)^{+}\right] .
$$

It is well known that the arbitrage-free relation between the forex spot rate and the forex forward rate are given by $F_{T}(t)=S(t) \frac{P_{f}(t, T)}{P_{d}(t, T)}$ where $P_{d}(t, T)$ and $P_{f}(t, T)$ denote the time $t$ values of domestic and foreign zero coupon bonds with maturity $T$ respectively. $\mathbf{E}^{P}[\cdot]$ denotes an expectation operator under $\operatorname{EMM(Equivalent}$ Martingale Measure) $P$ whose associated numeraire is the domestic zero coupon bond maturing at $T$.

For these pricing problems, a market model and a stochastic volatility model are applied to modeling interest rates' and the spot exchange rate's dynamics respectively.

We first define domestic and foreign forward interest rates as $f_{d j}(t)=\left(\frac{P_{d}\left(t, T_{j}\right)}{P_{d}\left(t, T_{j+1}\right)}-1\right) \frac{1}{\tau_{j}}$ and $f_{f j}(t)=$ $\left(\frac{P_{f}\left(t, T_{j}\right)}{P_{f}\left(t, T_{j+1}\right)}-1\right) \frac{1}{\tau_{j}}$ respectively, where $j=n(t), n(t)+1, \cdots, N, \tau_{j}=T_{j+1}-T_{j}$, and $P_{d}\left(t, T_{j}\right)$ and $P_{f}\left(t, T_{j}\right)$ denote the prices of domestic/foreign zero coupon bonds with maturity $T_{j}$ at time $t\left(\leq T_{j}\right)$ respectively; $n(t)=\min \left\{i: t \leq T_{i}\right\}$. We also define spot interest rates to the nearest fixing date denoted by $f_{d, n(t)-1}(t)$ and $f_{f, n(t)-1}(t)$ as $f_{d, n(t)-1}(t)=\left(\frac{1}{P_{d}\left(t, T_{n(t)}\right)}-1\right) \frac{1}{\left(T_{n(t)}-t\right)}$ and $f_{f, n(t)-1}(t)=\left(\frac{1}{P_{f}\left(t, T_{n(t)}\right)}-1\right) \frac{1}{\left(T_{n(t)}-t\right)}$. Finally, we set $T=T_{N+1}$ and will abbreviate $F_{T_{N+1}}(t)$ to $F_{N+1}(t)$ in what follows.

Under the framework of the asymptotic expansion in the standard cross-currency libor market model, we have to consider the following system of stochastic differential equations(henceforth called S.D.E.s) under the domestic terminal measure $P$ to price options. For detailed arguments on the framework of these S.D.E.s see $[20]$.

As for the domestic and foreign interest rates we assume forward market models; for $j=n(t)-$ $1, n(t), n(t)+1, \cdots, N$,

$$
\begin{aligned}
f_{d j}^{(\epsilon)}(t)= & f_{d j}(0)+\epsilon^{2} \sum_{i=j+1}^{N} \int_{0}^{t} g_{d i}^{0,(\epsilon)}(u)^{\prime} \gamma_{d j}(u) f_{d j}^{(\epsilon)}(u) d u+\epsilon \int_{0}^{t} f_{d j}^{(\epsilon)}(u) \gamma_{d j}^{\prime}(u) d W_{u}, \\
f_{f j}^{(\epsilon)}(t)= & f_{f j}(0)-\epsilon^{2} \sum_{i=0}^{j} \int_{0}^{t} g_{f i}^{0,(\epsilon)}(u)^{\prime} \gamma_{f j}(u) f_{f j}^{(\epsilon)}(u) d u+\epsilon^{2} \sum_{i=0}^{N} \int_{0}^{t} g_{d i}^{0,(\epsilon)}(u)^{\prime} \gamma_{f j}(u) f_{f j}^{(\epsilon)}(u) d u \\
& -\epsilon^{2} \int_{0}^{t} \sigma^{(\epsilon)}(u) \bar{\sigma}^{\prime} \gamma_{f j}(u) f_{f j}^{(\epsilon)}(u) d u+\epsilon \int_{0}^{t} f_{f j}^{(\epsilon)}(u) \gamma_{f j}^{\prime}(u) d W_{u},
\end{aligned}
$$

where $g_{d j}^{0,(\epsilon)}(t):=\frac{-\tau_{j} f_{d j}^{(\epsilon)}(t)}{1+\tau_{j} f_{d j}^{(\epsilon)}(t)} \gamma_{d j}(t), \quad g_{f j}^{0,(\epsilon)}(t):=\frac{-\tau_{j} f_{f j}^{(\epsilon)}(t)}{1+\tau_{j} f_{f j}^{(\epsilon)}(t)} \gamma_{f j}(t) ; x^{\prime}$ denotes the transpose of $x$ and $W$ is a $r$-dimensional standard Wiener process under the domestic terminal measure $P ; \gamma_{d j}(s), \gamma_{f j}(s)$ are $r$ dimensional vector-valued functions of time-parameter $s$; $\bar{\sigma}$ denotes a $r$-dimensional constant vector satisfying 
$\|\bar{\sigma}\|=1$ and $\sigma^{(\epsilon)}(t)$, the volatility of the spot exchange rate, is specified to follow a $\mathbf{R}_{++}$-valued general time-inhomogeneous Markovian process as follows:

$$
\sigma^{(\epsilon)}(t)=\sigma(0)+\int_{0}^{t} \mu\left(u, \sigma^{(\epsilon)}(u)\right) d u+\epsilon^{2} \sum_{j=1}^{N} \int_{0}^{t} g_{d j}^{0,(\epsilon)}(u)^{\prime} \omega\left(u, \sigma^{(\epsilon)}(u)\right) d u+\epsilon \int_{0}^{t} \omega^{\prime}\left(u, \sigma^{(\epsilon)}(u)\right) d W_{u}
$$

where $\mu(s, x)$ and $\omega(s, x)$ are functions of $s$ and $x$.

Finally, we consider the process of the forex forward $F_{N+1}(t)$. Since $F_{N+1}(t) \equiv F_{T_{N+1}}(t)$ can be expressed as $F_{N+1}(t)=S(t) \frac{P_{f}\left(t, T_{N+1}\right)}{P_{d}\left(t, T_{N+1}\right)}$, we easily notice that it is a martingale under the domestic terminal measure. In particular, it satisfies the following stochastic differential equation

$$
F_{N+1}^{(\epsilon)}(t)=F_{N+1}(0)+\epsilon \int_{0}^{t} \sigma_{F}^{(\epsilon)}(u)^{\prime} F_{N+1}^{(\epsilon)}(u) d W_{u}
$$

where

$$
\sigma_{F}^{(\epsilon)}(t):=\sum_{j=0}^{N}\left(g_{f j}^{0,(\epsilon)}(t)-g_{d j}^{0,(\epsilon)}(t)\right)+\sigma^{(\epsilon)}(t)
$$

\subsubsection{Numerical Examples}

We here specify our model and parameters, and confirm the effectiveness of our method in this cross-currency framework.

First of all, the processes of domestic and foreign forward interest rates and of the volatility of the spot exchange rate are specified. We suppose $r=4$, that is the dimension of a Brownian motion is set to be four; it represents the uncertainty of domestic and foreign interest rates, the spot exchange rate, and its volatility. Note that in this framework correlations among all factors are allowed. We also suppose $S(0)=100$.

Next, we specify a volatility process of the spot exchange rate in (37) with

$$
\mu(s, x)=\kappa(\theta-x), \omega(s, x)=\omega x,
$$

where $\theta$ and $\kappa$ represent the level and speed of its mean-reversion respectively, and $\omega$ denotes a volatility vector on the volatility. In this section the parameters are set as follows; $\epsilon=1, \sigma(0)=\theta=0.1$, and $\kappa=0.1$; $\omega=\omega^{*} \bar{v}$ where $\omega^{*}=0.3$ and $\bar{v}$ denotes a four dimensional constant vector given below.

We further suppose that initial term structures of domestic and foreign forward interest rates are flat, and their volatilities also have flat structures and are constant over time: that is, for all $j, f_{d j}(0)=f_{d}$, $f_{f j}(0)=f_{f}, \gamma_{d j}(t)=\gamma_{d}^{*} \bar{\gamma}_{d} 1_{\left\{t<T_{j}\right\}}(t)$ and $\gamma_{f j}(t)=\gamma_{f}^{*} \bar{\gamma}_{f} 1_{\left\{t<T_{j}\right\}}(t)$. Here, $\gamma_{d}^{*}$ and $\gamma_{f}^{*}$ are constant scalars, and $\bar{\gamma}_{d}$ and $\bar{\gamma}_{f}$ denote four dimensional constant vectors. Moreover, given a correlation matrix $\underline{C}$ among all four factors, the constant vectors $\bar{\gamma}_{d}, \bar{\gamma}_{f}, \bar{\sigma}$ and $\bar{v}$ can be determined to satisfy $\left\|\bar{\gamma}_{d}\right\|=\left\|\bar{\gamma}_{f}\right\|=\|\bar{\sigma}\|=\|\bar{v}\|=1$ and $V^{\prime} V=\underline{C}$ where $V:=\left(\bar{\gamma}_{d}, \bar{\gamma}_{f}, \bar{\sigma}, \bar{v}\right)$.

In this subsection, we consider four different cases for $f_{d}, \gamma_{d}^{*}, f_{f}$ and $\gamma_{f}^{*}$ as in Table 3. For correlations, four sets of parameters are considered: In the case "Corr.1", all the factors are independent: In "Corr.2", there exists only the correlation of -0.5 between the spot exchange rate and its volatility (i.e. $\bar{\sigma}^{\prime} \bar{v}=-0.5$ ) while there are no correlations among the others: In "Corr.3", the correlation between interest rates and the spot exchange rate are allowed while there are no correlations among the others; the correlation between domestic ones and the spot forex is $0.5\left(\bar{\gamma}_{d}^{\prime} \bar{\sigma}=0.5\right)$ and the correlation between foreign ones and the spot forex is $-0.5\left(\bar{\gamma}_{f}^{\prime} \bar{\sigma}=-0.5\right)$ : Finally in "Corr.4", more intricately correlated structure is considered; $\bar{\gamma}_{d}^{\prime} \bar{\sigma}=0.5$, $\bar{\gamma}_{f}^{\prime} \bar{\sigma}=-0.5$ between interest rates and the spot forex; and $\bar{\sigma}^{\prime} \bar{v}=-0.5$ between the spot forex and its volatility. It is well known that (both of exact and approximate)evaluation of the long-term options is a hard task in the case with complex structures of correlations such as in "Corr.3" or "Corr.4".

Lastly, we make an assumption that $\gamma_{d n(t)-1}(t)$ and $\gamma_{f n(t)-1}(t)$, volatilities of the domestic and foreign interest rates applied to the period from $t$ to the next fixing date $T_{n(t)}$, are equal to be zero for arbitrary 
Table 3: Initial domestic/foreign forward interest rates and their volatilities.

\begin{tabular}{|l|c|r|c|c|}
\cline { 2 - 5 } \multicolumn{1}{c|}{} & $f_{d}$ & \multicolumn{1}{c|}{$\gamma_{d}^{*}$} & $f_{f}$ & \multicolumn{1}{c|}{$\gamma_{f}^{*}$} \\
\hline case (i) & 0.05 & 0.12 & 0.05 & 0.12 \\
\hline case (ii) & 0.02 & 0.3 & 0.05 & 0.12 \\
\hline case (iii) & 0.05 & 0.12 & 0.02 & 0.3 \\
\hline case (iv) & 0.02 & 0.3 & 0.02 & 0.3 \\
\hline
\end{tabular}

$t \in\left[t, T_{n(t)}\right]$.

In Figure 1, we compare our estimations of the values of call and put options by an asymptotic expansion up to the fourth order to the benchmarks estimated by $10^{6}$ trials of Monte Carlo simulation which is discretized by Euler-Maruyama scheme with time step 0.05 and applied the Antithetic Variable Method. For the moneynesses(defined by $K / F_{N+1}(0)$ ) less than one, the prices of put options are shown; otherwise, the prices of call options are displayed. Detailed data obtained in this experiment, see Tabel 6-9 of [23].

As seen in this figure, in general the estimators show more accuracy as the order of the expansion increases. Especially, for the deep OTM options the fourth order approximation performs much better and is stabler than the approximation with lower orders.

\section{Concluding Remarks}

In this paper, we provided the general procedures for the explicit computation of conditional expectations necessary for practical computations of the asymptotic expansion method. Moreover, the alternative but equivalent calculation algorithm which computes the unconditional expectations directly instead of the conditional ones was developed. For simplicity and limitation of space, we focused on the simple case of Black-Scholes-type economy as in Section 2, which illustrated our key ideas. For further explanations in more general environment, see [23].

Finally, we exmained the accuracy of our approximation with high order expansions in the $\lambda$-SABR model and in the cross currency Libor market model with a stochastic volatility of the spot exchange rate, and confirmed satisfactory results in the both examples.

At the end of this section, we state our future plans: We will develop a similar result in the case with a jump component; we will also pursue an efficient method for the evaluation of multi-factor path-dependent or/and American derivatives. In fact, our proposed scheme can be applied to average options under a general setting of the underlying factors.

\section{References}

[1] Antonov, A. and Misirpashaev, T.[2009], "Projection on a Quadratic Model by Asymptotic Expansion with an Application to LMM Swaption," Working Paper.

[2] Andersen, L.B.G. and Hutchings, N.A.[2009], "Parameter Averaging of Quadratic SDEs With Stochastic Volatility," Working Paper.

[3] Fouque, J.-P., Papanicolaou, G. and Sircar, K. R.[1999], "Financial Modeling in a Fast Mean-reverting Stochastic Volatility Environment," Asia-Pacific Financial Markets, Vol. 6(1), pp.37-48.

[4] Fouque, J.-P., Papanicolaou, G. and Sircar, K. R.[2000], Derivatives in financial Markets with Stochastic Volatility, Cambridge University Press.

[5] Ikeda, N. and Watanabe, S. [1989], Stochastic Differential Equations and Diffusion Processes, Second Edition, North-Holland/Kodansha, Tokyo. 
[6] Kunitomo, N. and Takahashi, A. [1992], "Pricing Average Options," Japan Financial Review, Vol. 14, 1-20. (in Japanese).

[7] Kunitomo, N. and Takahashi, A. [2001], "The Asymptotic Expansion Approach to the Valuation of Interest Rate Contingent Claims," Mathematical Finance, Vol. 11, 117-151.

[8] Kunitomo, N. and Takahashi, A. [2003a], "On Validity of the Asymptotic Expansion Approach in Contingent Claim Analysis," Annals of Applied Probability Vol. 13-3, 914-952.

[9] Kusuoka S. and Osajima, Y. [2007], "A Remark on the Asymptotic Expansion of Density Function of Wiener Functionals," Preprint, Graduate School of Mathematical Sciences, the University of Tokyo.

[10] Labordere, P.H. [2005a], "A General Asymptotic Implied Volatility for Stochastic Volatility Models", cond-mat/0504317.

[11] Labordere, P.H. [2005b], "Solvable Local and Stochastic Volatility Models: Supersymmetric Methods in Option Pricing," Working Paper.

[12] Matsuoka, R. Takahashi, A. and Uchida, Y. [2004], "A New Computational Scheme for Computing Greeks by the Asymptotic Expansion Approach," Asia-Pacific Financial Markets, Vol.11, 393-430.

[13] Muroi, Y. [2005], "Pricing Contingent Claims with Credit Risk: Asymptotic Expansion Approach," Finance and Stochastics, Vol. 9(3), 415-427.

[14] Ninomiya, S. and Victoir, N. [2006], "Weak Approximation of Stochastic Differential Equations and Application to Derivative Pricing", Preprint.

[15] Nualart, D. [1995], "The Malliavin Calculus and Related Topics," Springer.

[16] Siopacha, M. and Teichmann, J.[2007], "Weak and Strong Taylor Methods for Numerical Solutions of Stochastic Differential Equations," Working paper.

[17] Takahashi, A. [1995], "Essays on the Valuation Problems of Contingent Claims," Unpublished Ph.D. Dissertation, Haas School of Business, University of California, Berkeley.

[18] Takahashi, A. [1999], "An Asymptotic Expansion Approach to Pricing Contingent Claims," Asia-Pacific Financial Markets, Vol. 6, 115-151.

[19] Takahashi, A. [2009], "On an Asymptotic Expansion Approach to Numerical Problems in Finance," Selected Papers on Probability and Statistics, pp. 199-217, 2009, American Mathematical Society.

[20] Takahashi, A. and Takehara, K.[2007], "Pricing Currency Options with a Market Model of Interest Rates under Jump-Diffusion Stochastic Volatility Processes of Spot Exchange Rates," Asia-Pacific Financial Markets, Vol.14 , pp. 69-121.

[21] Takahashi, A. and Takehara, K.[2008a], "Fourier Transform Method with an Asymptotic Expansion Approach: an Applications to Currency Options," International Journal of Theoretical and Applied Finance, Vol. 11(4), pp. 381-401.

[22] Takahashi, A. and Takehara, K. [2008b], "A Hybrid Asymptotic Expansion Scheme: an Application to Currency Options," Working paper, CARF-F-116, the University of Tokyo, http://www.carf.e.utokyo.ac.jp/workingpaper/

[23] Takahashi, A., Takehara, K. and Toda, M. [2009], "Computation in an Asymptotic Expansion Method," Working paper, CARF-F-149, the University of Tokyo, http://www.carf.e.u-tokyo.ac.jp/workingpaper/

[24] Takahashi, A. and Yamada, T. [2008], "An Asymptotic Expansion with Push Down Malliavin Weights," Preprint.

[25] Takahashi, A. and Yoshida, N. [2004], "An Asymptotic Expansion Scheme for Optimal Investment Problems," Statistical Inference for Stochastic Processes, Vol. 7-2, 153-188. 
[26] Takahashi, A. and Yoshida, N. [2005], "Monte Carlo Simulation with Asymptotic Method," The Journal of Japan Statistical Society, Vol. 35-2, 171-203.

[27] Watanabe, S. [1987], "Analysis of Wiener Functionals (Malliavin Calculus) and its Applications to Heat Kernels," The Annals of Probability, Vol. 15, 1-39.

[28] Yoshida, N. [1992a], "Asymptotic Expansion for Small Diffusions via the Theory of MalliavinWatanabe," Probability Theory and Related Fields, Vol. 92, 275-311.

[29] Yoshida, N. [1992b], "Asymptotic Expansions for Statistics Related to Small Diffusions," The Journal of Japan Statistical Society, Vol. 22, 139-159. 
Figure 1: Graphs of comparisons of estimators by the third- and fourth-order asymptotic expansion and Monte Carlo simulations in Corr.1-4, with a ten-year maturity. Squares denote the differences between the third-order estimators and Monte Carlo estimators; circles denote those between the fourth-order ones and Monte Carlo ones. These differences are defined by (the approximate value by our asymptotic expansion) (the estimator by Monte Carlo simulations).

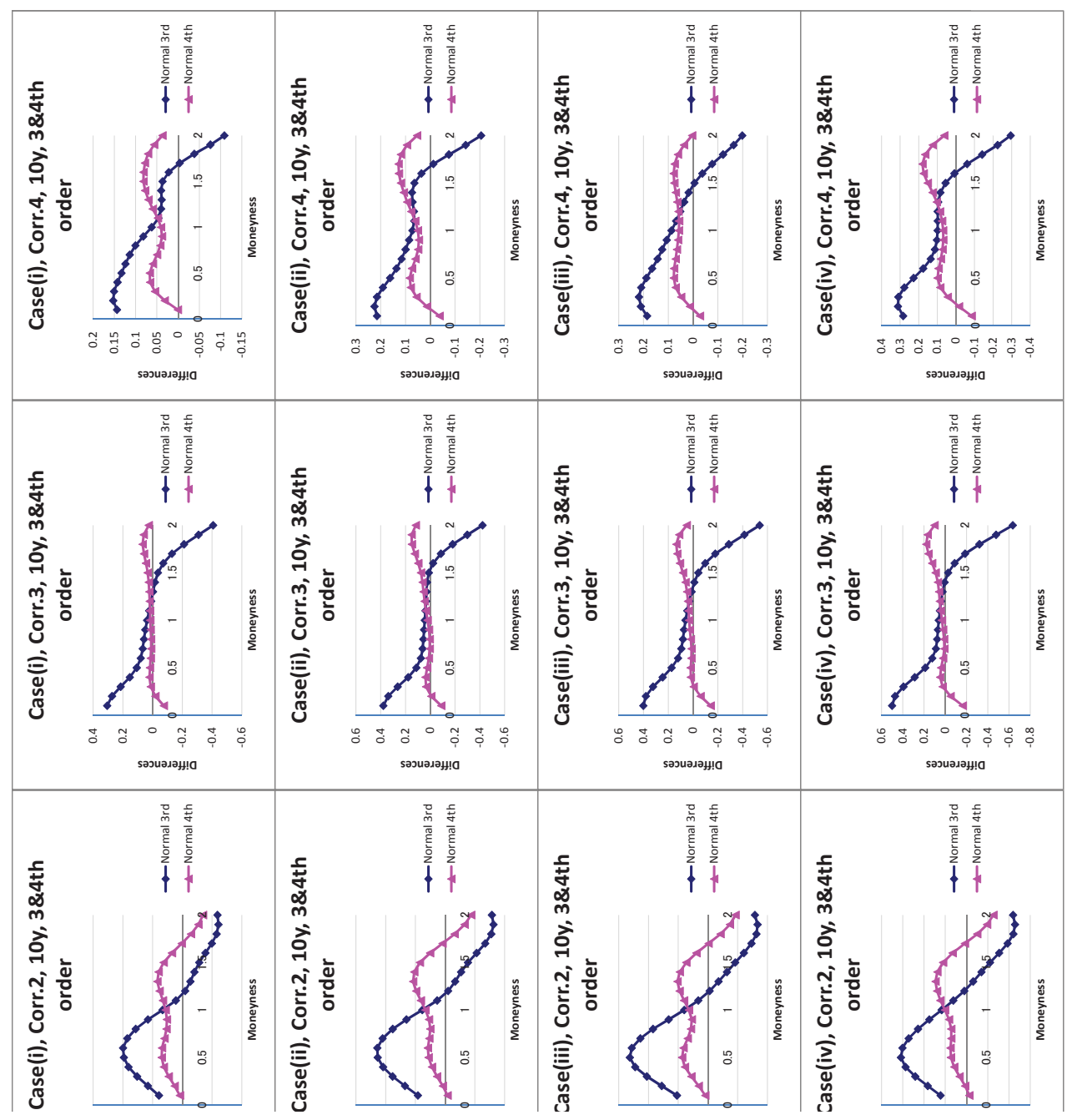

